\title{
Serotonin syndrome due to duloxetine and tramadol use in an older patient
}

\author{
Olayinka A. Ogundipe*
}

Department of Medicine of the Elderly, Royal Infirmary of Edinburgh, 51 Little France Crescent, Edinburgh, Scotland

Received: 24 April 2020

Revised: 13 May 2020

Accepted: 15 May 2020

\section{*Correspondence:}

Dr. Olayinka A. Ogundipe,

Email: ola_ayodele@hotmail.com

Copyright: $\odot$ the author(s), publisher and licensee Medip Academy. This is an open-access article distributed under the terms of the Creative Commons Attribution Non-Commercial License, which permits unrestricted non-commercial use, distribution, and reproduction in any medium, provided the original work is properly cited.

\begin{abstract}
This case report describes a 92-year old woman presenting with acute confusion and agitation. She was initially diagnosed as having a hyperactive delirium. However, based on the presence of additional and evolving features of twitchiness, reduced coordination, palpitations and headaches, the diagnosis was re-evaluated. The clinical presentation was subsequently recognised as being that of the serotonin syndrome. In this instance, the serotonin syndrome was judged to have arisen from the concurrent use of duloxetine and tramadol. Duloxetine is an antidepressant with serotonergic properties. Tramadol is an analgesic agent with weak opiate agonist receptor effects, and also exerts reuptake inhibition of noradrenaline and serotonin. The patient's polypharmacy was reviewed, and alongside other general supportive care measures, her symptoms and signs resolved within 48 hours. This report serves as a clinical reminder on the potential pitfalls of polypharmacy in older patients. Delirium is a common presentation in older patients, and on occasions, clearly establishing the underlying causes or risk factors may prove challenging or even elusive. The report prompts clinicians to bear in mind that the presentation and diagnosis of the serotonin syndrome requires a high index of suspicion, and that patients may present atypically. In support of pharmacovigilance reporting, two scales of causality assessment are employed in this case review. The application of these systems exemplifies their potential in promoting and enhancing objectivity when clinicians report suspected adverse drug reactions (ADRs) noted in routine clinical practice.
\end{abstract}

Keywords: Adverse drug reaction, Delirium, Duloxetine, Pharmacovigilance, Serotonin and noradrenaline reuptake inhibitor, Serotonin syndrome, Tramadol

\section{INTRODUCTION}

The serotonin syndrome presents clinically as symptoms and signs occurring in association, and often related to the use of medications with serotonergic properties or effects. ${ }^{1}$ These may present as a result of adverse drug reactions (ADRs) to single agents, or as adverse drug interactions between two or more medications. ${ }^{1,2}$

The syndrome often manifests as a three-fold presentation: an altered mental status; autonomic system dysfunction; and neurological symptoms. ${ }^{1}$ However, the condition may also present atypically, and may thereby go unrecognised and/or may not be managed appropriately. The latter scenario escalates the risk that the clinical features of the serotonin syndrome may evolve into potentially life-threatening complications. ${ }^{2}$

Polypharmacy related adverse reactions or side-effects, and resultant delirium are commonly noted occurrences in the clinical management of older patients. ${ }^{3}$ 
This report offers a clinical reminder that polypharmacy may cause an older patient to develop the serotonin syndrome, and that the presentation may mimic hyperactive delirium. The report highlights the need for clinicians to be familiar with the range of symptoms and signs of the serotonin syndrome, and also stresses the need to adopt a high index of suspicion.

\section{CASE REPORT}

A 92-year-old Caucasian lady was admitted to hospital with reports of feeling shaky, anxious and being acutely confused over a few days. She had also recently complained of headaches, occasional palpitations and mild breathlessness.

She had a medical history of mixed Alzheimer's and vascular dementia, bilateral macular degeneration and possible associated Charles-Bonnet syndrome (visual hallucinations in the context of visual impairment). Her multi-morbidity also included a history of bilateral carpal tunnel syndrome, chronic mixed musculoskeletal and neuropathic pains, depression, gastritis, hypertension, ischaemic heart disease and osteoporosis. Her regular medications were adcal-D3 one tablet twice daily, amlodipine $5 \mathrm{mg}$ once daily, chlorpromazine $25 \mathrm{mg}$ once at night, duloxetine hydrochloride $30 \mathrm{mg}$ once daily, galantamine MR $24 \mathrm{mg}$ once daily, omeprazole $20 \mathrm{mg}$ once daily, paracetamol $500 \mathrm{mg}$ four times a day, pregabalin $150 \mathrm{mg}$ twice daily, tramadol hydrochloride MR $100 \mathrm{mg}$ twice daily, quetiapine $25 \mathrm{mg}$ nocte, and zopiclone $7.5 \mathrm{mg}$ nocte. She had no drug allergies but her medical records noted gastrointestinal intolerance to aspirin and clopidogrel.

On admission, examination noted a temperature of $36.6^{\circ} \mathrm{C}$, blood pressure $132 / 69 \mathrm{mmHg}$, pulse $80 \mathrm{bpm}$, respiratory rate $24 / \mathrm{min}$, and oxygen saturations of $92 \%$ breathing air. Capillary blood sugar was 5.2. An abbreviated mental test score (AMTS) was 8 out of 10 , and she was disorientated to place and circumstances. There was no meningism and her speech was normal. Pupils were small but not constricted. She exhibited twitchiness and reduced coordination, but otherwise further central nervous system examination was unremarkable. She had mild fine bi-basal inspiratory crackles. Heart sounds were unremarkable with no murmurs. Abdominal examination was normal.

Investigations revealed that her blood count, renal, liver and thyroid function tests, as well as C-reactive protein (CRP) were all unremarkable. A resting 12-lead electrocardiogram (ECG) demonstrated sinus rhythm, occasional atrial premature complexes, normal PR and QTc intervals, and voltage criteria suggestive of left ventricular hypertrophy. A chest X-ray noted age-related unfolding of the thoracic aorta, and minor basal changes suggestive of a degree of lung fibrosis.

Table 1: Naranjo ADRs algorithm. ${ }^{15}$

Questionnaire applied to index case report

Are there previous conclusive reports on this reaction?

Yes (+1) No (0) Do not know or not done (0)

Did the adverse event appear after the suspected drug was given?

Yes (+2) No (-1) Do not know or not done (0)

Did the adverse reaction improve when the drug was discontinued or a specific antagonist was given?

Yes (+1) No (0) Do not know or not done (0)

Did the adverse reaction appear when the drug was re-administered?

Yes (+2) No (-2) Do not know or not done (0)

Are there alternative causes that could have caused the reaction?

Yes (-1) No (+2) Do not know or not done (0)

Did the reaction reappear when a placebo was given?

Yes (-1) No (+1) Do not know or not done (0)

Was the drug detected in any body fluid in toxic concentrations?

Yes (+1) No (0) Do not know or not done (0)

Was the reaction more severe when the dose was increased or less severe when the dose was decreased?

Yes (+1) No (0) Do not know or not done (0)

Did the patient have a similar reaction to the same or similar drugs in any previous exposure?

Yes (+1) No (0) Do not know or not done (0)

Was the adverse event confirmed by any objective evidence?

Yes (+1) No (0) Do not know or not done (0)

$>9=$ definite ADR

$5-8=$ probable ADR

$1-4=$ possible ADR

$0=$ doubtful ADR

In this case, a total score of 7 translates to a classification of a 'probable' adverse drug reaction (ADR). 
Table 2: WHO-UMC causality categories. ${ }^{18}$

\begin{tabular}{|c|c|}
\hline Causality term & Assessment criteria* \\
\hline \multirow{5}{*}{ Certain } & Event or laboratory test abnormality, with plausible time relationship to drug intake \\
\hline & Cannot be explained by disease or other drugs \\
\hline & Response to withdrawal plausible (pharmacologically, pathologically) \\
\hline & $\begin{array}{l}\text { Event definitive pharmacologically or phenomenologically (i.e. an objective and specific medical } \\
\text { disorder or a recognized pharmacological phenomenon) }\end{array}$ \\
\hline & Re-challenge satisfactory, if necessary \\
\hline \multirow{4}{*}{ Probable/likely } & Event or laboratory test abnormality, with reasonable time relationship to drug intake \\
\hline & Unlikely to be attributed to disease or other drugs \\
\hline & Response to withdrawal clinically reasonable \\
\hline & Re-challenge not required \\
\hline \multirow{3}{*}{ Possible } & Event or laboratory test abnormality, with reasonable time relationship to drug intake \\
\hline & Could also be explained by disease or other drugs \\
\hline & Information on drug withdrawal may be lacking or unclear \\
\hline \multirow[t]{2}{*}{ Unlikely } & $\begin{array}{l}\text { Event or laboratory test abnormality, with a time to drug intake that makes a relationship improbable (b } \\
\text { not impossible) }\end{array}$ \\
\hline & Disease or other drugs provide plausible explanations \\
\hline \multirow{3}{*}{$\begin{array}{l}\text { Conditional/ } \\
\text { unclassified }\end{array}$} & Event or laboratory test abnormality \\
\hline & More data for proper assessment needed, or \\
\hline & Additional data under examination \\
\hline \multirow{3}{*}{$\begin{array}{l}\text { Unassessable/ } \\
\text { unclassifiable }\end{array}$} & Report suggesting an adverse reaction \\
\hline & Cannot be judged because information is insufficient or contradictory \\
\hline & Data cannot be supplemented or verified \\
\hline
\end{tabular}

The admitting doctor diagnosed a hyperactive delirium in view of her acutely confused state, and noting the risk factor of known dementia. Following senior clinical review, the possibility was considered that the symptoms and signs were related to the serotonin syndrome. The rationale for this suspicion was the significant polypharmacy, and the presence of additional features (i.e. over and above the noted acute confusion/delirium) e.g. the tremulousness/twitchiness, headaches, and palpitations.

Based on the clinical findings, the collateral history was explored in greater depth with the patient's daughter. This indicated that the patient was not taking 'over-thecounter' or complementary medicines. The collateral established that the patient's previous occupational history, as well as more recent social and travel histories were unremarkable. She was a non-smoker and tee-total. On direct questioning, her daughter also conveyed a concern that the patient might have taken additional unmonitored doses of ('as required') oral tramadol for her analgesia requirements. The latter concern raised the possibility of inadvertent or unintentional tramadol overdose in the context of the patient's memory problems due to dementia. Neither her family nor the admitting clinical team had noted any signs to suggest the presence of distressing visual hallucinations, and there had been no reports of diarrhoea.

In arriving at the clinical diagnosis of serotonin syndrome, emphasis was placed on the concurrent use of duloxetine and tramadol, with note taken of the possibility of (accidental) tramadol overuse or overdose.
Her extensive polypharmacy was rationalised in a staged manner, and the impact on her symptoms were monitored. Priority was given to stopping the duloxetine completely, and concurrently reducing the maintenance dose of the tramadol MR to $50 \mathrm{mg}$ twice daily. As the tramadol was a longstanding prescription for her chronic pain, the author decided not to stop it abruptly to avoid precipitating an acute opioid withdrawal. This strategy of a phased medication review resulted in a notable improvement to her symptoms within 48 hours. Specifically, the features that improved rapidly were the delirium, headaches, palpitation, tremulousness/shakiness, and the feelings of being anxious.

In the 24 hours prior to her discharge, the doses of some of her other drugs were reduced or discontinued. For e.g., the author stopped the low dose chlorpromazine (to reduce the risks of extra-pyramidal side-effects and falls). The author also reduced the dose of pregabalin to $125 \mathrm{mg}$ twice daily (with further plans to 'wean down' slowly over time to reduce the risks of side-effects from acute withdrawal). In addition, the omeprazole was reduced to $10 \mathrm{mg}$ once daily (to reduce the risks of clostridium difficile infection, iron malabsorption, hyponatraemia, and osteoporosis that may accompany its long-term use).

As she had been taking most of the medications for many years, 'intermediate term' plans were communicated in the typed hospital discharge letter. The discharge letter to her primary care physician/general practitioner recommended a plan to progressively reduce her other polypharmacy. The advice was for the pregabalin, tramadol, zopiclone, and quetiapine to be reviewed in a 
sequential and monitored manner to reduce the risk of withdrawal effects.

She was successfully discharged from hospital after a further 24-hour period of unremarkable clinical observation i.e. after a cumulative 72-hour hospital admission.

\section{DISCUSSION}

The serotonin syndrome is the occurrence of clinical symptoms and signs in correlation with one another, often in association with the use of serotonergic agents, and/or arising from the interactions between different medications with serotonergic effects. ${ }^{1}$ This often arises as a consequence of an adverse drug reaction (ADR) to a single drug, or potentially as adverse drug interactions occurring between medications. ${ }^{1,2}$

\section{Some associations of the serotonin syndrome}

The syndrome has been associated with the initiation of new drugs, or with concurrent use of other medications that have serotonergic effects.,5 Other associations include high cumulative doses (e.g. as legitimately prescribed doses, accidental usage, or in overdose scenarios) and following prolonged periods of therapy. ${ }^{6,7}$ The syndrome may also manifest in the presence of conditions that alter drug pharmacokinetics e.g. the presence of concomitant chronic kidney disease (CKD). ${ }^{8}$

\section{Duloxetine, tramadol and the serotonin syndrome}

Duloxetine is an antidepressant with serotonergic properties, and is classified as a selective serotonin and noradrenaline reuptake inhibitor (SSNRI). ${ }^{9}$ Tramadol has analgesic properties derived from partial mu opioid agonist receptor effects. ${ }^{10}$ Tramadol also exerts reuptake inhibition towards both noradrenaline and serotonin. The fact that duloxetine and tramadol share some neurotransmitter pathways may be one reason for their association with precipitating the serotonin syndrome.

The mechanism of causation of the serotonin syndrome may be linked to pharmacokinetic and/or pharmacodynamic mechanisms of various culprit drugs. In this patient, it could be postulated that a pharmacokinetic effect was less likely. For example, she had biochemically normal liver and renal function. The latter would support arguments for a preserved ability to undertake hepatic metabolism, and also to eliminate a medication such as tramadol that undergoes predominantly renal excretion. A postulate for the principal mechanism of the serotonin syndrome in this patient might favour a pharmacodynamic association or synergy; in this instance due to drug-drug interactions between the duloxetine and tramadol.

The symptoms and signs of the serotonin syndrome can range from being mild up to a potentially life-threatening condition or complication. The features of the syndrome are typically characterised by a tri-fold manifestation of altered mental status; autonomic dysfunction or symptoms; and neurological symptoms or neuromuscular hyperactivity. The foregoing may variably present as agitation or agitated states, tremulousness or tremors, hyperreflexia, ataxia, altered sensorium/confusion/delirium, hyperthermia, shivering, and loose stools or diarrhoea.

However, it is important to stress that the triad presentation described above is not always present. Clinicians need to be aware that patients may present without features from a particular group of these symptoms and signs. A further significant point is that even when a tri-fold cluster of symptoms and signs does occur in a specific patient, these need not manifest nor evolve in a linear/sequential order. Given the aforementioned wide range of features, and the abovementioned caveats, it becomes even more evident that some patients may present atypically.

\section{Ageing, polymorbidity, polypharmacy, delirium, and the serotonin syndrome}

Unfortunately, issues such as polymorbidity, polypharmacy and delirium are all commonly encountered during the provision of clinical care to older patients presenting to hospital. ${ }^{3,11}$

The index case presented with signs suggestive of a hyperactive delirium (i.e. as an acutely confused state with associated agitation). However, she was noted to have other features that are less typical for delirium (e.g. tremulousness, reduced coordination, headaches and palpitations). The presence of these additional findings and taking note of her polypharmacy factored into the clinical reassessment and the revised diagnosis of the serotonin syndrome.

In evaluating this patient, other differentials were considered but deemed unlikely. These include viral encephalitis (no fever; no meningism; normal CRP) and neuroleptic malignant syndrome - NMS (no fever; no hypertonia; no suggestion of abrupt withdrawal of exogenous agents like dopaminergic agonists or modulators; and no recent major changes to her drugs).

The principles of management are based around having a pre-existing awareness of the serotonin syndrome, and taking preventative measures when prescribing drugs with serotonergic effects. ${ }^{1}$ Early and appropriate case recognition is also important, as is undertaking ongoing reviews of polypharmacy linked to the syndrome. ${ }^{3,11}$ The approach taken, and rapidity of drug withdrawals or dose reductions are dependent upon an individual patient's clinical context.

In this case, the patient's rapid symptom improvement within a 48-hour period of making changes to relevant 
medications (specifically, stopping the duloxetine and concurrently reducing the dose of tramadol MR) was also supportive of the clinical diagnosis.

Other general supportive clinical measures that were considered included assessing and managing tachycardia or cardiac arrhythmia. In this case, the palpitations settled without requiring anti-arrhythmic agents. Some patients require antipyretics but this patient was afebrile at the time of presentation. Alternative analgesia may be required for some patients, especially if full withdrawal of tramadol is necessitated. Further measures that the author undertook included the optimisation of her hydration status, and serial monitoring to exclude the co-existence of any significant electrolyte or acid-base imbalance (e.g. metabolic acidosis). The latter state could aggravate cardiac arrhythmias in the context of the serotonin syndrome. In addition, the author excluded the presence of any significant metabolic disorders (e.g. hypoxia and hypo/hyperglycaemia) which could potentially worsen acutely confused states.

In this case, the author judged it clinically unnecessary and even potentially inappropriate to undertake a medication re-challenge. In arriving at this decision, the author noted the patient's advanced age, physical frailty, and the clinical risks vs benefits. The author also acknowledged the fact that the medical literature records some previously documented cases of SSRIs and tramadol (either singly or jointly) causing the serotonin syndrome. . $2,4,6-8,12-14^{-1}$

\section{Applying two causality assessment scales to the index case}

Many ADRs are categorised as 'suspected' reports, i.e. rather than as 'certain or definite' events. The presence of confounding factors often underlies this outcome. Evaluating this report using causality scales offers further objectivity and transparency in the pharmacovigilance reporting of the suspected ADR. Applying the Naranjo Scale to the index report derives a score of 7 (Table 1) and this equates to a 'probable' ADR classification. ${ }^{15-17}$ Using the WHO-UMC methodology (Table 2) translates to a comparable 'probable/likely' ADR classification. ${ }^{18}$

\section{CONCLUSION}

As patients age, it is not uncommon for polypharmacy to accompany the presence of multi-morbidity. As exemplified by this case, such polymorbidity may be physical (e.g. chronic pain from osteoarthritis and carpal tunnel neuropathy), and/or mental health related (e.g. dementia and depression).

This report serves as a clinical reminder of a potential pitfall of polypharmacy in older patients. The report highlights the value of clinicians undertaking reviews of their patients' medicines with a view to identifying any possible side-effects or ADRs.
More specifically, the report also illustrates the need to consider the serotonin syndrome as a differential diagnosis of hyperactive delirium in an older person presenting with other unusual clinical findings. Consequently, it is pertinent that clinicians maintain an awareness of, and have a high index of suspicion for the syndrome. Furthermore, clinicians who care for older patients need to be increasingly able to recognise the wide-ranging, and sometimes atypical presentations of the serotonin syndrome.

Funding: No funding sources

Conflict of interest: None declared

Ethical approval: Not required

\section{REFERENCES}

1. Buckley NA, Dawson AH, Isbister GK. Serotonin syndrome. BMJ. 2014;348:g1626.

2. Kumar BN, Shah R, Grover S. Serotonin syndrome while switching antidepressants. Indian J Psychiatry. 2011;53:372.

3. Hein C, Forgues A, Piau A, Sommet A, Vellas B, Nourhashémi. Impact of polypharmacy on occurrence of delirium in elderly emergency patients. JAMDA Online. 2014;15(11):850.e11-850.e15.

4. George TP, Godleski LS. Possible serotonin syndrome with trazodone addition to fluoxetine. Biol Psychiatry. 1996;39:384-5.

5. Houlihan DJ. Serotonin syndrome resulting from coadministration of tramadol, venlafaxine and mirtazapine. Annals Pharmacother. 2004;38:411-3.

6. Canan F, Korkmaz U, Kocer E, Onder E, Yildirim S, Ataoglu A. Serotonin syndrome with paroxetine overdose: a case report. Prim Care Companion J Clin Psychiatry. 2008;10:165-7.

7. Nayyar N. Serotonin syndrome associated with sertraline, trazodone and tramadol abuse. Indian $\mathrm{J}$ Psychiatry. 2009;51:68.

8. Raj R, Kumar R, Sidhu BS, Yakhami S. Serotonin syndrome due to fluoxetine and tramadol in renal impaired patient. Int J Basic Clin Pharmacol. 2014;3:227-9.

9. British National Formulary. National Institute for Health and Care Excellence. CNICE 2020. Available at: https://bnf.nice.org.uk/drug/duloxetine.html. Accessed on $13^{\text {th }}$ May 2020.

10. British National Formulary. National Institute for Health and Care Excellence. (C) NICE 2020. Available at: https://bnf.nice.org.uk/drug/tramadolhydrochloride.html. Accessed on $13^{\text {th }}$ May 2020.

11. Poeschla BD, Bartle P, Hansen KP. Serotonin syndrome associated with polypharmacy in the elderly. Gen Hosp Psychiatry. 2011;33(3):301.e9-11.

12. Shakoor MT, Ayub S, Ahad A, Ayub Z. Transient serotonin syndrome caused by concurrent use of tramadol and selective serotonin reuptake inhibitor. Am J Case Rep. 2014;15:562-4. 
13. Takeshita J, Litzinger MH. Serotonin syndrome associated with tramadol. Prim Care Companion J Clin Psychiatry. 2009;11:273.

14. Liu, PT, Argento, V, Skudlarska, B, Blagodatny, M. Serotonin syndrome in an octogenarian after switch from fluoxetine to duloxetine. J Am Geriat Society. 2009;57:2384-4.

15. Naranjo CA, Busto U, Sellers EM, Sandor P, Ruiz I, Roberts EA, et al. A method for estimating the probability of adverse drug reactions. Clin Pharmacol Ther. 1981;30(2):239-45.

16. Busto U, Naranjo CA, Sellers EM. Comparison of two recently published algorithms to assess the probability of adverse drug reactions. $\mathrm{Br} \mathrm{J}$ Clin Pharmacol. 1982;13(2):223-7.

17. Kramer MS, Leventhal JM, Hutchinson TA, Feinstein AR. An algorithm for the operational assessment of adverse drug reactions. I. Background, description, and instructions for use. JAMA. 1979;242(7):623-32.

18. The use of the WHO-UMC system for standardized case causality assessment. Uppsala Monitoring Centre. Available at: https://www.who.int/ medicines/areas/quality_safety/safety_efficacy/WHO causality_assessment.pdf Accessed on $13^{\text {th }}$ May 2020.

Cite this article as: Ogundipe OA. Serotonin syndrome due to duloxetine and tramadol use in an older patient. Int J Basic Clin Pharmacol 2020;9:1124-9. 